\title{
MÉTODOS GEOESTATÍSTICOS NO ESTUDO DA RESISTÊNCIA DO SOLO À PENETRAÇÃO EM TRILHA DE TRÁFEGO DE TRATORES NA COLHEITA DE MADEIRA $^{1}$
}

\author{
Julião Soares de Souza Lima², Paulo César Oliveira ${ }^{3}$, Rone Batista de Oliveira ${ }^{3}$ e Alexandre Cândido
} Xavier $^{2}$

\begin{abstract}
RESUMO - Este trabalho teve como objetivo avaliar a resistência do solo à penetração (RSP) em seção transversal à trilha de tráfego dos tratores "Feller-buncher" e "Skidder" na colheita de madeira, utilizando-se as técnicas de geoestatística na determinação da dependência espacial e interpolação por krigagem. Ajustaram-se modelos de semivariogramas para determinação da dependência espacial antes do tráfego e depois do corte e do arraste. O coeficiente de variação da RSP na trilha diminuiu com o tráfego. Os resultados indicaram moderada dependência espacial da RSP antes e depois do corte, ajustando-se o modelo esférico. O modelo linear ajustou-se às semivariâncias após o arraste da madeira, indicando, nesse caso, a necessidade de se trabalhar com uma malha maior para representar a continuidade do atributo. A metodologia geoestatística mostrou ser boa ferramenta para estimar valores em pontos não amostrados e na construção dos mapas de isolinhas da RSP pelo método de interpolação por krigagem.
\end{abstract}

Palavras-chave: Variabilidade espacial, semivariograma e colheita mecanizada.

\section{GEOSTATISTIC METHODS USED IN THE STUDY OF SOIL PENETRATION RESISTANCE IN TRACTOR TRAFFIC TRAIL DURING WOOD HARVESTING}

\begin{abstract}
This work was carried out to evaluate soil penetration resistance (SPR) in a transversal section of the traffic trail of the tractors Feller-buncher and Skidder during wood harvesting. Geostatistic techniques were used to determine spatial dependence and kriging interpolation. Semivariogram models were fit to determine spatial dependence before traffic and after cutting and skidding. The SPR variation coefficient in the trail reduced with traffic. The result showed a moderate SPR spatial dependence in the soil section before and after cutting, with the spherical model being adjusted. The linear model was adjusted to semivariance after wood skidding, indicating the need to use a larger grid to represent the continuity of the attribute. The geostatistic methodology was found to be a good tool to estimate the values in non-sampled points and in SPR contour map building.
\end{abstract}

Keywords: Spatial variability, semivariograms and mechanized harvesting.

\footnotetext{
${ }^{1}$ Recebido em 08.03.2007 e aceito para publicação em 22.08.2008.

${ }^{2}$ Departamento de Engenharia Rural da Universidade Federal do Espírito Santo, Alegre-ES. E-mail: <juliaosslima@ cca.ufes.br>.

${ }^{3}$ Programa de Pós-Graduação em Produçaõ Vegetal da UFES.
} 


\section{INTRODUÇÃO}

Os efeitos causados ao solo florestal nas operações mecanizadas podem ser minimizados do ponto de vista operacional dos tratores, adotando-se módulos de colheita sem comprometer a sua produtividade, fazendo tráfego em menores áreas dentro do talhão, redução das trilhas de tráfego e transporte de menores cargas. Alternativas estão sendo adotadas em algumas empresas, como: uso de tratores florestais com rodados de esteiras, pneus duplos, pneus com maiores dimensões com baixa pressão interna de inflagem e alta flutuação (BPAF) e rodados em Tandem, com o intuito de proporcionar menor compactação mecânica do solo.

A compactação de solos florestais difere dos solos agrícolas em função das espécies cultivadas, do tamanho e dos ciclos das culturas e das máquinas utilizadas. Mas as forças causadoras da compactação nos dois tipos de solos ocorrem em decorrência do intenso uso de máquinas (GREACEN e SANDS, 1980).

Segundo Balastreire (1987), a resistência do solo à penetração (RSP) é uma propriedade dinâmica do solo identificada como a reação do solo à ação de uma força aplicada através de uma ponteira cônica. A RSP pode ser influenciada pela densidade, pelo teor de água e pelo tipo de solo.

Estudos experimentais têm mostrado que a resistência do solo aumenta com a elevação da densidade e diminui com o incremento no teor de água do solo. A resistência determinada por penetrômetros de cone tem sido usada ao longo de muitos anos com várias aplicações, em muitos campos de pesquisas, por ser de fácil uso, rápido diagnóstico do nível de compactação em várias profundidades e operacionalmente econômico, podendo ser afetada pelo diâmetro da base, ângulo e aspereza da superfície do cone (PERUMPRAL, 1987).

A variabilidade espacial da RSP foi estudada por Mercante et al. (2003) em áreas com diferentes manejos e em profundidades, utilizando um penetrógrafo de haste, com ângulo de cone de $30^{\circ}$. Foram efetuados os cálculos das semivariâncias experimentais pelo estimador de Matheron com o ajuste pelos modelos teóricos. Neste estudo, os resultados indicaram que a RSP apresentou variabilidade espacial em relação aos diferentes manejos e profundidades do solo.

A variabilidade espacial de algumas características do solo vem sendo uma das preocupações de vários pesquisadores. Os procedimentos iniciais baseavamse na estatística clássica e utilizavam grandes quantidades de dados amostrais, visando caracterizar ou descrever a distribuição espacial do atributo estudado. Por estatística clássica, entende-se aquela que se utilizam parâmetros como média e desvio-padrão para representar um fenômeno e se baseia na hipótese principal de que as variações de um local para outro são aleatórias (CAMARGO, 1997).

Segundo Vieira (2000), alguns trabalhos mostram que somente a informação dada pela variância seria insuficiente para explicar um fenômeno estudado. Para tal, seria necessário levar em consideração a distância entre as observações. A partir daí surge o conceito da geoestatística que leva em consideração a localização geográfica e a dependência espacial.

Este trabalho teve como objetivo estudar a viabilidade das técnicas de geoestatística na variabilidade espacial da resistência do solo à penetração em seções transversais à trilha de tráfego, antes e depois da colheita, em povoamento de eucalipto, para construção de mapas de isolinhas para esse atributo.

\section{MATERIAL E MÉTODOS}

O estudo foi conduzido em um povoamento florestal constituído de Eucalyptus camaldulensis Dehnh. $x$ Eucalyptus grandis W. Hill ex Maiden, no espaçamento de 3,0 x 1,5 m, com uma produtividade média de 144,7 st/ha, implantado em um Latossolo Vermelho-Amarelo, textura muito argilosa, com a seguinte composição granulométrica média: argila $(74,5 \%)$, silte $(6,0 \%)$, areia fina $(4,5 \%)$ e areia grossa $(15,3 \%)$, em topografia plana no Município de Itamarandiba, MG. A precipitação média anual é de $1.150 \mathrm{~mm}$, com clima da região subtropical úmido e subúmido. A densidade e o teor de água no solo foram determinados à profundidade de $0 \mathrm{a} 45 \mathrm{~cm}$, apresentando valores médios de $1,0 \mathrm{~kg} \mathrm{dm}^{-3}$ e $23,0 \%$, respectivamente.

A RSP foi determinada no período de julho de 1998, com um penetrógrafo de mola de registro contínuo, equipado com uma ponta em forma de cone e ângulo de vértice de 30 graus, sendo o valor da leitura registrado em função da profundidade de penetração até $45 \mathrm{~cm}$, conforme descrito por Lima (1998) e Mercante et al. (2003).

O levantamento da RSP transversalmente na trilha de tráfego foi realizado em três condições: antes do tráfego (ATR), depois do corte (DCORTE) e depois 
do arraste do feixe de fuste semi-suspenso (DARRASTE). Foram realizadas leituras espaçadas em $30 \mathrm{~cm}$, totalizando 11 pontos, com leituras em profundidade de 5 em 5 $\mathrm{cm}$ até $45 \mathrm{~cm}$, obtendo-se 99 valores por seção. As leituras da RSP na segunda e terceira seções foram espaçadas de $10 \mathrm{~cm}$ da primeira seção construída (antes do tráfego), conforme metodologia descrita por Lima (2003). Com os dados obtidos, construiu-se uma malha retangular de 9 (profundidades) x 11 (pontos), conforme Figura 1.

O sistema de colheita adotado foi o de fuste (tree length), desenvolvendo-se as atividades de corte com um trator derrubador-amontoador ("Feller-Buncher") com um cabeçote de corte equipado com disco dentado, rodados de pneus diagonais e massa total de 13.525 $\mathrm{kg}$. O desgalhamento e o destopamento foram realizados por motosserristas. Para o arraste do feixe de fuste foi utilizado um trator florestal arrastador, "Skidder", com $16.245 \mathrm{~kg}$ de massa, chassi articulado, com pneus de baixa pressão e alta flutuação (BPAF) e adaptado com uma pinça hidráulica traseira para o abraçamento e levantamento do feixe de fuste no arraste semi-suspenso.

A estatística descritiva foi utilizada para estudar a tendência e dispersão dos dados em cada seção transversal, bem como a sua forma de distribuição, antes e depois de cada operação. Para a verificação da distribuição de tendência dos pontos em cada malha, utilizou-se a construção do post-plot com divisão em quartis, com os valores mostrados em cores diferentes.

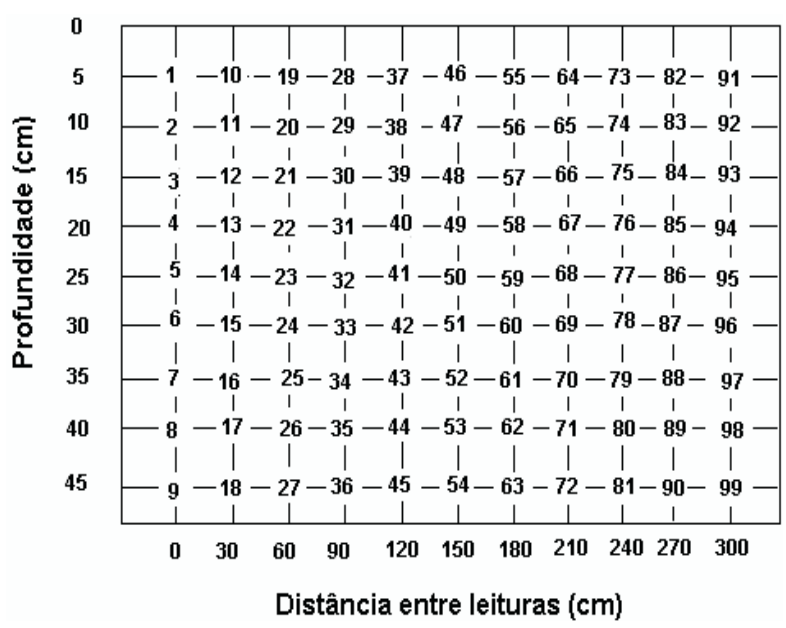

Figura 1 - Malha da seção transversal na trilha de tráfego dos tratores.

Figure 1 -Transversal section grid in the tractor traffic trail.
A dependência espacial foi avaliada pelas técnicas da geoestatística com base nas pressuposições de estacionaridade da hipótese intrínseca, em que a variância da diferença depende somente da distância entre os pontos amostrais e não da posição em que eles se encontram; e pela análise de semivariogramas, utilizandose o software $\mathrm{GS}^{+}$(ROBERTSON, 1998). As semivariâncias determinadas em função da distância entre os pontos foram calculadas pela Equação 1.

$$
{ }^{*}(h)=\frac{1}{2 N} * \sum_{i=1}^{N(h)}\left[Z\left(x_{i}\right)-\left(x_{i}+h\right)\right]^{2}
$$

em que $N(h)$ é o número de pares experimentais separados por uma distância $\mathrm{h} ; Z\left(x_{i}\right)$ é o valor determinado em cada ponto amostrado; e $Z\left(x_{i}+\mathrm{h}\right)$ é o valor medido num ponto mais uma distância h. O gráfico de gama asterisco $\left(\gamma^{*}\right)$ em função da distância $(h)$ é denominado semivariograma.

Outro parâmetro do semivariograma que foi determinado é o alcance (a) que indica o limite da dependência espacial do atributo em estudo, ou seja, determinações realizadas a distâncias maiores que o alcance tem distribuição espacial aleatória e, por isso, são independentes entre si, podendo ser aplicada a estatística clássica (SILVA et al., 2003). No entanto, determinações realizadas a distâncias menores que o alcance são correlacionadas umas com as outras, o que permite que se façam interpolações para espaçamentos menores que os amostrados.

Em seguida, os semivariogramas foram escalonados pela variância dos dados, de acordo com Vieira et al. (1997); com isso o efeito pepita, que é a descontinuidade do semivariograma na origem, tornou-se automaticamente uma fração do patamar, facilitando as interpretações e comparações entre semivariogramas nas diferentes condições, bem como observações da presença do mesmo padrão de variabilidade espacial, uma vez que este assume valores em uma escala padronizada. O semivariograma escalonado $\gamma^{*} e$ foi calculado pela equação 2 .

$$
\gamma^{*} e=\frac{\gamma_{i}^{*}(h)}{S^{2}}
$$

em que $i$ vai de 1 até o número de variáveis medidas, e $S^{2}$ é o valor da variância dos dados de cada situação.

Testaram-se semivariogramas dos tipos esférico, exponencial, linear e gaussiano. A escolha dos modelos

R. Árvore, Viçosa-MG, v.32, n.5, p.931-938, 2008 
teóricos foi realizada, observando-se a soma do quadrado dos resíduos (SQR), coeficiente de determinação $\left(\mathrm{R}^{2}\right)$ e, posteriormente, o coeficiente de correlação obtido pela técnica da validação cruzada (SILVA et al., 2003).

Para análise do grau de dependência espacial (GDE) da RSP foi utilizada a relação $C_{1} /\left(C_{0}+C_{1}\right)$, classificada segundoZimback (2001) como forte (GDE $\geq 0,75)$, moderada $(0,25 \leq \mathrm{GDE}<0,75)$ e baixa dependência $(\mathrm{GDE}<0,25)$.

Conhecendo o semivariograma do atributo e apresentando dependência espacial entre os dados, fezse a interpolação pelo método da krigagem ordinária no software $\mathrm{GS}^{+}$. Nesse método, pontos próximos da posição a serem interpolados levam maiores pesos que os mais distantes. Esse estimador nada mais é que uma média móvel ponderada (VIEIRA, 2000).

A estimativa por krigagem ordinária segue a equação3.

$$
Z *\left(x_{i}\right)=\sum \lambda_{i} \cdot Z\left(x_{i}\right)
$$

em que $\lambda_{i}$ são pesos de cada valor medido, $Z\left(x_{i}\right)$ é o valor medido e $Z^{*}\left(x_{i}\right)$ é o valor estimado através do método de krigagem.

Os pesos $\lambda$ que são obtidos pelo método de multiplicadores de Lagrange, associados à equação de estimação e à exigência de que a esperança dos erros seja igual a zero e a variância de estimação seja mínima, fazem com que a variância de krigagem seja a menor variância entre todos os processos de interpolação (GUIMARÃES, 2000).

\section{RESULTADOS E DISCUSSÃO}

Os dados da análise descritiva no estudo da resistência do solo à penetração (RSP) estão apresentados no Quadro 1.

Analisando os dados, observou-se que a média e a mediana não apresentaram valores próximos nas três condições de tráfego, mostrando, como confirmado pelo teste de Shapiro-Wilks a 5\% de probabilidade, de que os dados não apresentaram distribuição próxima da normal. O coeficiente de variação (CV) da RSP exibiu diminuição na condição do solo antes do tráfego para depois do arraste do feixe de fuste pelo "Skidder" na trilha. Isso pode ser visto pela menor amplitude entre os dados depois da operação de arraste. O tráfego dos tratores na trilha proporcionou incremento na sua compactação, como indicam os valores médios da RSP. Os dados apresentam distribuição assimétrica, porém com curtose negativa, evidenciando distribuição platicúrtica com maior dispersão dos dados em relação à média.

A RSP apresentou alto valor nas três condições de estudo. Segundo Tormena (2002), valores de RSP entre 2 e 3 MPa são considerados limitantes do desenvolvimento radicular, porém o valor é considerado limitante, segundo Dedecek (2005), acima de 3 Mpa para o sistema radicular do eucalipto. Cabe ressaltar que, após a colheita, a área passaria por uma reforma do povoamento.

O tráfego na trilha afetou a RSP até $45 \mathrm{~cm}$ de profundidade, mostrando ser até essa profundidade maiores os distúrbios causados ao solo pela realização das operações nas condições do estudo e que nessa profundidade os valores se mantiveram próximos, como observado na Figura 2.

Analisando a seção transversal da RSP, na trilha, antes do tráfego com os valores agrupados por quartis pelo post-plot (Figura 2a), observou-se que existe concentração de valores do quarto quartil na região inferior à direita, com 26,3\% dos maiores valores da RSP. A mediana dos dados apresentou um valor 3,41 $\mathrm{MPa}$, indicando solo compactado. Notou-se que a RSP foi aumentando com o tráfego, como era de se esperar, atingindo o máximo valor a $45 \mathrm{~cm}$ de profundidade. Essas figuras ilustram o comportamento anisotrópico dos dados da RSP nas condições de estudo, com uma clara tendência de variar com a profundidade.

Quadro 1 - Dados da análise descritiva da resistência do solo à penetração antes do tráfego e depois do corte e do arraste Table 1 -Descriptive analysis data of soil penetration resistance (SPR) before traffic and after cutting and skidding

\begin{tabular}{|c|c|c|c|c|c|c|c|c|}
\hline \multirow[t]{2}{*}{ Condição } & Média & Mediana & \multirow[t]{2}{*}{$\mathrm{S}$} & Min & Máx. & \multicolumn{3}{|c|}{ Coeficiente } \\
\hline & \multicolumn{2}{|c|}{$(\mathrm{MPa})$} & & \multicolumn{2}{|c|}{ (MPa) } & $\mathrm{CV}$ & $\mathrm{C}_{\mathrm{s}}$ & $\mathrm{C}_{\mathrm{k}}$ \\
\hline$\overline{\mathrm{ATR}}$ & 3,60 & 3,41 & 1,89 & 0,56 & 6,74 & 52,6 & 0,05 & $-0,99$ \\
\hline DCORTE & 4,58 & 4,36 & 1,23 & 2,46 & 6,74 & 26,8 & 0,21 & $-1,12$ \\
\hline DARRASTE & 5,00 & 5.31 & 0,85 & 2,94 & 6,74 & 17,0 & $-0,29$ & $-0,22$ \\
\hline
\end{tabular}

ATR = antes do tráfego; DCORTE = depois do corte; DARRASTE = depois do arraste; S = desvio-padrão; Min - valor mínimo; Máx = valor máximo; $C V=$ coefic. de variação; Assim = coefic. de assimetria; e Curt = coefic. de curtose.

R. Árvore, Viçosa-MG, v.32, n.5, p.931-938, 2008 
(a)

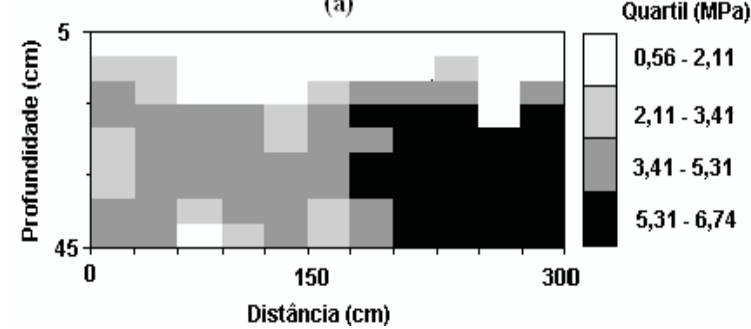

(b)

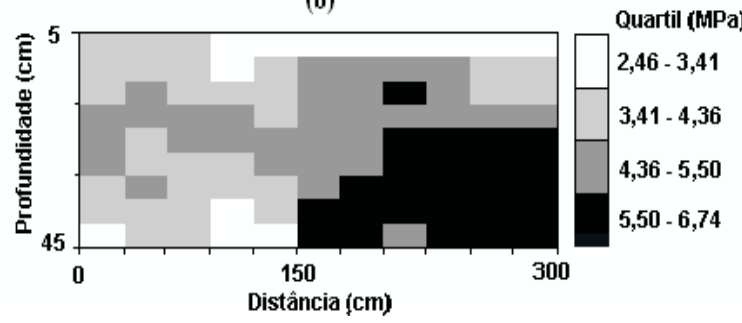

(c)

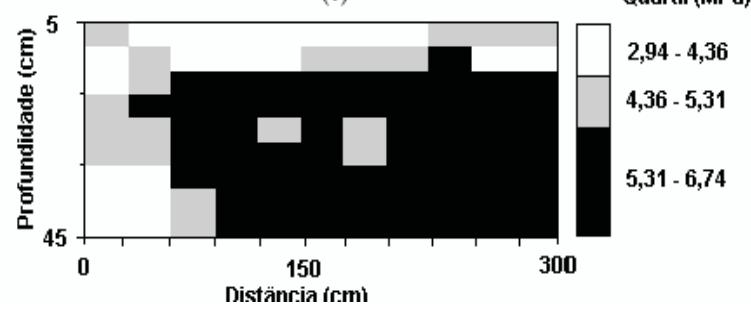

Figura 2 - Pos-plot da seção transversal da resistência do solo à penetração na trilha: antes do tráfego (a), depois do corte (b) e depois do arraste (c).

Figure 2-Post-plot of the transversal section of the soil penetration resistance in the trail: before traffic $(a)$, after cutting and $(c)$ after skidding.

Os maiores valores da RSP foram detectados na seção após o arraste das toras, como observado na Figura 2c. A pressão dos pneus e do feixe de fuste semi-suspenso deslizando sobre a trilha proporcionou maior aproximação entre os valores no sentido da superfície e, conseqüentemente, maior compactação do solo. Segundo Machado et al. (2004), muitos fenômenos naturais apresentaram atributos cujos dados espaciais tenderam a ser similares ou dissimilares quanto mais próximos da posição se encontravam.

O software $\mathrm{GS}^{+}$utilizado na análise permitiu construir o semivariograma médio, considerando-se todas as direções da seção, conforme mostrado na Figura 3.

Os parâmetros dos modelos teóricos ajustados aos semivariogramas experimentais escalonados estão no Quadro 2.

A RSP apresentou ajuste do modelo esférico (ESF) para antes do tráfego e depois do corte. Isso indica que os semivariogramas explicam a maior parte da variância dos dados experimentais. Depois do arraste, ajustouse o modelo linear para os dados da RSP. Nesse caso, indica-se grande capacidade de dispersão dos dados na seção estudada, mostrando que a profundidade da malha e, ou, a distância entre as leituras foram insuficientes para estudar a variabilidade espacial da RSP nessa situação, como discutido por Vieira (2000).

O efeito pepita $\left(\mathrm{C}_{0}\right)$ variou de 0,47 a 0,61 , refletindo no grau de dependência espacial que oscilou de 0,50 a 0,64 , indicando moderada correlação espacial entre os pontos vizinhos.

Os alcances de dependência espacial foram de 228 e $192 \mathrm{~cm}$ para ATR e DCORTE, respectivamente, indicando maior continuidade espacial horizontal até essas distâncias nas trilhas. Os valores de RSP aumentaram na horizontal e se aproximaram da superfície em função da pressão dos rodados no solo no tráfego do "Feller-buncher" (Figura 4b).

Quadro 2 - Dados dos semivariogramas escalonados da resistência do solo à penetração na seção transversal antes e depois do corte e do arraste

Table 2 - Data of the scaled semivariograms of soil penetration resistance in the transversal section before and after cutting and skidding

\begin{tabular}{lcccccc}
\hline Condição & Modelo & $\mathrm{C}_{0}$ & $\mathrm{C}_{0}+\mathrm{C}_{1}$ & $\mathrm{a}$ & $\mathrm{IDE}$ & $\mathrm{R}^{2}$ \\
\hline ATR & ESF & 0,61 & 1,24 & 228 & 0,50 & 0,88 \\
DCORTE & ESF & 0,47 & 1,26 & 192 & 0,63 & 0,94 \\
DARRASTE & LIN & 0,56 & 1,58 & - & 0,64 & 0,90 \\
\hline
\end{tabular}

$\mathrm{ESF}=$ modelo esférico $; \mathrm{EXP}=$ modelo exponencial $; \mathrm{LIN}=$ modelo linear; $\mathrm{C}_{0}=$ efeito pepita; $\mathrm{C}_{0}+\mathrm{C}_{1}=$ patamar; $\mathrm{a}=$ alcance da dependência espacial $(\mathrm{cm}) ; \mathrm{IDE}=$ índice de dependência espacial; $\mathrm{R}^{2}=$ coeficiente de determinação do modelo; GDE = grau de dependência espacial; e Mod. = nível de dependência moderada. 
(a)

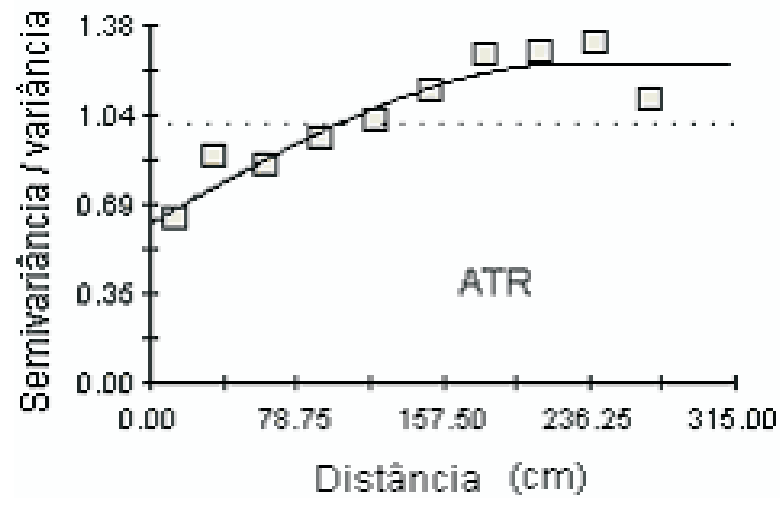

(b)

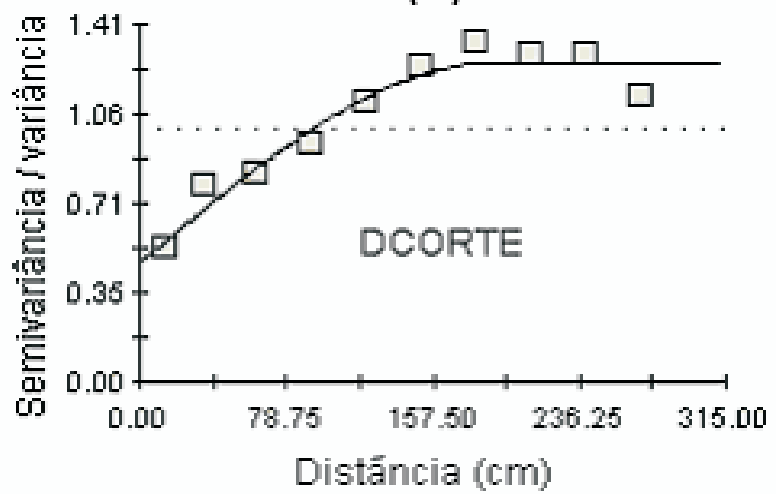

(c)

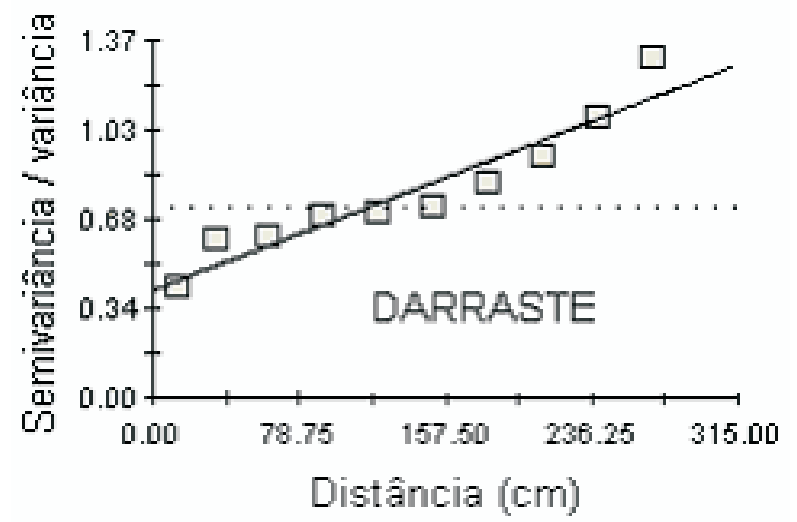

Figura 3 - Semivariogramas escalonados da resistência do solo à penetração nas seções transversais da trilha: antes do tráfego (ATR) (a), depois do corte (DCORTE) (b) e depois do arraste (DARRASTE) (c).

Figure 3-Scaled semivariograms of soil penetration resistance in the transversal sections of the trail: before traffic, after cutting and skidding.

R. Árvore, Viçosa-MG, v.32, n.5, p.931-938, 2008
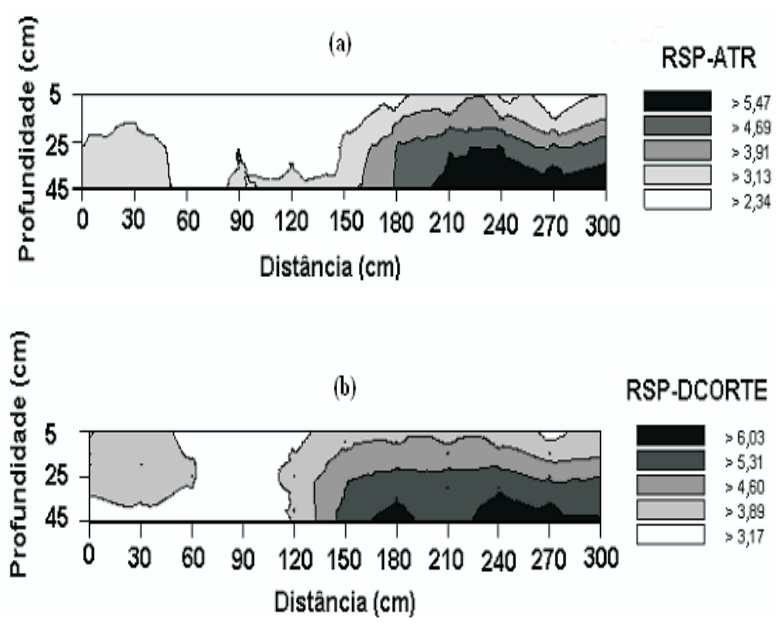

Figura 4 - Mapa de isolinhas da RSP na seção transversal da trilha: antes do tráfego (ATR) (a) e depois do corte (DCORTE) (b).

Figure 4-Soil penetration resistance contour map in the transversal section of the trail before traffic (a) and after cutting $(b)$.

Após a definição da dependência espacial da RSP antes do tráfego (ATR) e depois do corte (DCORTE), realizou-se a krigagem ordinária dos dados (Figura 4ab) para obtenção dos mapas temáticos dessas seções transversais, estimando valores em locais não medidos e com menor variância da estimada, o que é uma característica desse método (GUIMARÃES, 2000). Cabe ressaltar que o método de interpolação utilizado não permite a construção, com sucesso, do mapa temático da RSP após o arraste do feixe de fuste (DARRASTE), por não apresentar definição do patamar no semivariograma, conforme mostrado na Figura $3 \mathrm{c}$.

Essas figuras mostram alterações significativas no comportamento da RSP, no perfil do solo, com o tráfego dos tratores florestais utilizados na colheita de madeira. Essas mudanças são mais marcantes nos valores próximos à superfície, quando da realização da operação de corte, comparativamente com a situação do solo antes do tráfego. Isso indica maiores alterações após a passagem do primeiro trator florestal na trilha, conforme mostrado por Lima (1998) e Fernandes e Souza. (2003). Tal fato é explicado pela pressão dos rodados sobre o solo, proporcionando maior diminuição dos vazios nessa operação e também, depois, pela carga aplicada ao solo no deslizamento do feixe de fuste semisuspenso no arraste. 


\section{CONCLUSÕES}

- O coeficiente de variação da resistência do solo à penetração na trilha de tráfego diminuiu com o aumento da compactação provocada pelos tratores florestais.

- O tráfego do "Feller-buncher" proporcionou incremento na resistência do solo à penetração no sentido da superfície, com menor variabilidade entre os valores na horizontal e maior em profundidade.

- A resistência do solo à penetração apresentou dependência espacial entre as leituras, e valores menores que o alcance foram correlacionados, contribuindo para a melhor estimativa de valores em locais não medidos.

- Os métodos geoestatísticos aplicados mostramse viáveis para o estudo da variabilidade espacial da resistência do solo à penetração (RSP) em seções transversais à linha de tráfego.

\section{REFERÊNCIAS}

BALASTREIRE, L. A. Máquinas agrícolas. São Paulo: Manolo, 1987. 307p.

\section{CAMARGO, E. C. G. Desenvolvimento, implementação e teste de procedimentos geoestatísticos (krigeagem) no sistema de processamento de informações georreferenciadas (Spring). 1997. $123 \mathrm{f}$. Dissertação (Mestrado) - Instituto Nacional Pesquisa Espaciais, São José dos Campos, 1997.}

DEDECEK, R. A.; GAV, J. L. Influência da compactação do solo na produtividade da rebrota de eucalipto. Revista Árvore, v.29, n.3., p.383-390, 2005.

FERNANDES, H. C.; SOUZA, A. P. Compactação de um latossolo vermelho causada pelo tráfego do "forwarder". Revista Árvore, v.27, n.3, p.279-284, 2003.

GREACEN, E. L.; SANDS, R. Compaction of forest soils a review. Gleis Osmond:

Australian Journal of Soil Research, v.18, n.2, p.163-89,1980.
GUIMARÃES, E C. Variabilidade espacial de atributos de um Latossolo Vermelho escuro textura argilosa da região do cerrado, submetido ao plantio direto e ao plantio convencional. 2000. 92f. Tese (Doutorado) Universidade Estadual de Campinas, Campinas, 2000 .

LIMA, J. S. S. Avaliação da força de arraste, compactação do solo e fatores ergonômicos dos tratores florestais "Feller-Buncher" e "Skidder" utilizados na colheita de madeira. 1998. 128f. Tese (Doutorado) - Universidade Federal de Viçosa, Viçosa, MG,1998.

LIMA, J. S. S. et al. Estudo do comportamento da resistência do solo à penetração em trilha de tráfego na colheita de madeira. In: SIMPÓSIO BRASILEIRO SOBRE COLHEITAE TRANSPORTE FLORESTAL, 5., 2001, Porto Seguro. Anais... Viçosa, MG: Sociedade de Investigações Florestais, 2001. p.281-289.

PERUMPRAL, J. V. Cone penetrometer application - A review. Transactions of the American Society of Agricultural Engineering, v.30, n.4, p.939-944, 1987

MACHADO, P. L. O.; BERNARDI, A. C.; SILVA, A. C. Agricultura de precisão para o manejo da fertilidade do solo em sistema de plantio direto. Rio de Janeiro: Embrapa, 2004. 209p.

MERCANTE, E.; URIBE-OPOZO, M. A.; SOUZA, E. G. Variabilidade espacial e temporal da resistência mecânica do solo à penetração em áreas com e sem manejo químico localizado. Revista Brasileira Ciência do Solo, v.27, n.6. p.1149-1159, 2003.

ROBERTSON, G. P. GS ${ }^{+}$: Geostatistics for the environmental sciences $-\mathrm{GS}^{+} \mathrm{User}$ 's Guide. Plainwell: Gamma Design Software, 1978. 152p.

SILVA, V. R. et al. Variabilidade espacial das características químicas do solo e produtividade de milho em um Argissolo Vermelho Amarelo Distrófico Arénico. Revista Brasileira Ciência do Solo, v.27, n.6, p.1013-1020, 2003.

R. Árvore, Viçosa-MG, v.32, n.5, p.931-938, 2008 
TORMENA, C. A. A compactação do solo em agroecossistemas agrícolas. In: REUNIÃO BRASILEIEA DE MANEJO E CONSERVAÇÃO DO SOLO E DA ÁGUA, 14., 2002, Viçosa. Anais... Viçosa, MG: sociedade Brasileira de Ciência do Solo, 2002. 4p. CD ROM.

VIEIRA, S. R. Variabilidade espacial de argila, silte e atributos químicos em uma parcela experimental de um Latossolo roxo de Campinas (SP). Bragantia, v.56, n.1, p.1-13, 1997.
VIEIRA, S. R. Geoestatística em estudos de variabilidade espacial do solo. In. NOVAES, R. F.; ALVAREZ V., V. H.; SCHAEFER, C. E G. R. Tópicos em ciências do solo. Viçosa, MG: Sociedade Brasileira de Ciência do Solo, 2000. v.1. p.2-54.

ZIMBACK, C. R. L. Análise espacial de atributos químicos de solos para fins de mapeamento da fertilidade. 2001. 114f. Tese (Livre-Docência em Levantamento do solo e fotopedologia) - FCA-UNESP, Botucatu, 2001. 